University of Nebraska - Lincoln

DigitalCommons@University of Nebraska - Lincoln

\title{
Structure/function analyses of human serum paraoxonase (HuPON1) mutants designed from a DFPase-like homology model
}

\author{
David T. Yeung \\ US Army Medical Research Institute of Chemical Defense, Aberdeen Proving Ground, MD \\ Denis Josse \\ Centre de Recherches du Service de Sante' des Arme'es, La Tronche \\ James D. Nicholson \\ US Army Medical Research Institute of Chemical Defense, Aberdeen Proving Ground, MD \\ Akhil Khanal \\ University of Delaware \\ Christopher W. McAndrew \\ University of Delaware \\ See next page for additional authors
}

Follow this and additional works at: https://digitalcommons.unl.edu/usarmyresearch

Part of the Operations Research, Systems Engineering and Industrial Engineering Commons

\footnotetext{
Yeung, David T.; Josse, Denis; Nicholson, James D.; Khanal, Akhil; McAndrew, Christopher W.; Bahnson, Brian B.; Lenz, David E.; and Cerasoli, Douglas M., "Structure/function analyses of human serum paraoxonase (HuPON1) mutants designed from a DFPase-like homology model" (2004). US Army Research. 68.

https://digitalcommons.unl.edu/usarmyresearch/68
}

This Article is brought to you for free and open access by the U.S. Department of Defense at DigitalCommons@University of Nebraska - Lincoln. It has been accepted for inclusion in US Army Research by an authorized administrator of DigitalCommons@University of Nebraska - Lincoln. 


\section{Authors}

David T. Yeung, Denis Josse, James D. Nicholson, Akhil Khanal, Christopher W. McAndrew, Brian B. Bahnson, David E. Lenz, and Douglas M. Cerasoli 


\title{
Structure/function analyses of human serum paraoxonase (HuPON1) mutants designed from a DFPase-like homology model
}

\author{
David T. Yeung ${ }^{\mathrm{a}}$, Denis Josse ${ }^{\mathrm{b}}$, James D. Nicholson ${ }^{\mathrm{a}}$, Akhil Khanal ${ }^{\mathrm{c}}$, \\ Christopher W. McAndrew ${ }^{c}$, Brian J. Bahnson ${ }^{c}$, David E. Lenz ${ }^{\mathrm{a}}$, Douglas M. Cerasoli ${ }^{\mathrm{a}, *}$ \\ a Biochemical Pharmacology Branch, Pharmacology Division, US Army Medical Research Institute of Chemical Defense, \\ Aberdeen Proving Ground, MD 21010-5400, USA \\ ${ }^{\mathrm{b}}$ Département de Toxicologie, Centre de Recherches du Service de Santé des Armées, La Tronche 38702, France \\ ${ }^{\mathrm{c}}$ Department of Chemistry and Biochemistry, University of Delaware, Newark, DE 19716, USA
}

Received 2 June 2004; received in revised form 3 August 2004; accepted 4 August 2004

Available online 23 August 2004

\begin{abstract}
Human serum paraoxonase (HuPON1) is a calcium-dependent enzyme that hydrolyzes esters, including organophosphates and lactones, and exhibits anti-atherogenic properties. A few amino acids have been shown to be essential for the enzyme's arylesterase and organophosphatase activities. Until very recently, a three-dimensional model was not available for HuPON1, so functional roles have not been assigned to those residues. Based on sequence-structure alignment studies, we have folded the amino acid sequence of HuPON1 onto the sixfold $\beta$-propeller structure of squid diisopropylfluorophosphatase (DFPase). We tested the validity of this homology model by circular dichroism (CD) spectroscopy and site-directed mutagenesis. Consistent with predictions from the homology model, CD data indicated that the structural composition of purified HuPON1 consists mainly of $\beta$-sheets. Mutants of HuPON1 were assayed for enzymatic activity against phenyl acetate and paraoxon. Substitution of residues predicted to be important for substrate binding (L69, H134, F222, and C284), calcium ion coordination (D54, N168, N224, and D269), and catalytic mechanism of HuPON1 (H285) led to enzyme inactivation. Mutants F222Y and $\mathrm{H} 115 \mathrm{~W}$ exhibited substrate-binding selectivity towards phenyl acetate and paraoxon, respectively. The homology model presented here is very similar to the recently obtained PON1 crystal structure, and has allowed identification of several residues within the enzyme active site. (C) 2004 Elsevier B.V. All rights reserved.
\end{abstract}

Keywords: Arylesterase; Paraoxonase; HuPON1; $\beta$-Propeller; Paraoxon; Organophosphatase

\section{Introduction}

Organophosphorus (OP) nerve agents are highly toxic compounds that rapidly inhibit acetylcholinesterase [1]. Current treatments for OP poisoning are effective in preventing lethality from OP exposure, but do not prevent the toxic effects and incapacitation observed in animals and likely to occur in humans [2]. Consequently, current research efforts are focused on the development of a bioscavenger [3] to remove OP compounds from circulation before they can

\footnotetext{
* Corresponding author. Tel.: +1 410436 1338; fax: +1 4104368377

E-mail address: douglas.cerasoli@us.army.mil (D.M. Cerasoli).
}

reach their physiological target [4]. Anti-OP bioscavengers should be specific and efficient, function under physiological conditions, and not produce any deleterious pharmacological side effects in the absence of OP exposure [2,5-7]. Human paraoxonase 1 (HuPON1) is a serum protein that is associated with high-density lipoprotein (HDL) in vivo $[8,9]$. HuPON1 exhibits esterase and anti-oxidative activities that may be related to its anti-atherogenic properties $[8,10]$. Additionally, the level of HuPON1 in circulation has been reported to correlate with resistance to OPs [11-14], suggesting that PON1 acts as an in vivo bioscavenger [6]. Although the enzyme has a naturally low level of catalytic activity towards OPs, upon optimization it could be a useful and efficient catalytic bioscavenger. 
Our current efforts are focused on the design of HuPON1 variants with enhanced activity against OPs. Previous work in our laboratory has demonstrated that an enzyme's catalytic efficiency can be altered by sitedirected mutagenesis. Specifically, a genetically engineered $\mathrm{G} 117 \mathrm{H}$ (in which the glycine found at amino acid residue 117 of the wild-type enzyme is replaced with a histidine) human butyrylcholinesterase (HuBuChE) mutant [15] is catalytically active against OPs such as echothiophate and paraoxon [16], in contrast to wild-type $\mathrm{HuBuChE}$, which is irreversibly inhibited by OPs. We estimate that a 10 -fold increase in the catalytic activity of HuPON1 against OPs would be sufficient to provide substantial in vivo protection against poisoning by chemical warfare nerve agents [17].

HuPON1 is a $43-k D a$ glycosylated protein [18] comprised of 354 amino acids. Prior experiments utilized groupspecific reagents followed by site-directed mutagenesis to identify residues essential for enzyme activity. Results showed that residues E53, D54, D169, D183, E195, D269, D279, and C284, histidine residues H115, H134, $\mathrm{H} 155, \mathrm{H} 243$, and H285 and tryptophan residue W281 are critical for HuPON1 arylesterase and organophosphatase activities [17,19]. It has also been shown that cysteine residues 42 and 353 form a disulfide bond in the mature protein, and are essential for PON1 secretion and catalytic activity $[19,20]$. The polymorphic Q/R192 residue also plays a role in HuPON1's hydrolysis of OP compounds $[21,22]$. Based on amino acid sequence alignments between HuPON1, diisopropylfluorophosphatase (DFPase) from $L$. vulgaris [23], and mouse Senescence Marker Protein (SMP30) [24], we proposed that HuPON1 and DFPase share a similar structure in which six blade-like regions of $\beta$-pleated sheets are arranged radially around a central core (a sixfold $\beta$-propeller pleated structure) (see Fig. 1; Ref. [22]).

Knowledge of the three-dimensional structure of HuPON1 is required for the rational design of mutants that may enhance catalytic efficiency and stability. Although a three-dimensional X-ray crystallographic structure of a PON1 variant, generated by directed evolution and gene shuffling of human, mouse, rat and rabbit PON1 genes, has recently been reported [20], the aim of the present study was to critically evaluate the proposed three-dimensional homology model of the HuPON1 enzyme based on the structure of DFPase [22] and to identify residues important for enzymatic function. Our strategy to obtain that information included circular dichroism (CD) spectroscopy and site-directed mutagenesis studies. Amino acid residues predicted from our model to be important for catalytic activity were substituted with residues exhibiting different properties (e.g., altered charge, polarity, or size). HuPON1 has been shown to have enzymatic activities against multiple classes of substrates [9,12,21,25-28]; expressed mutant HuPON1 enzymes were tested against two classes of substrates (arylesters and OPs). Both the recently reported

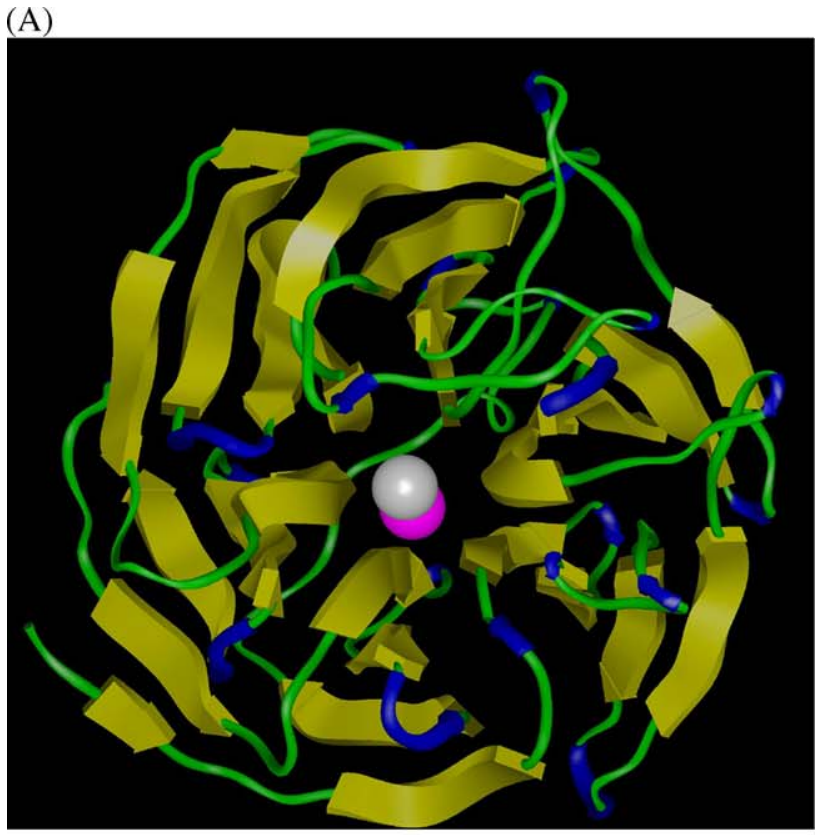

(B)

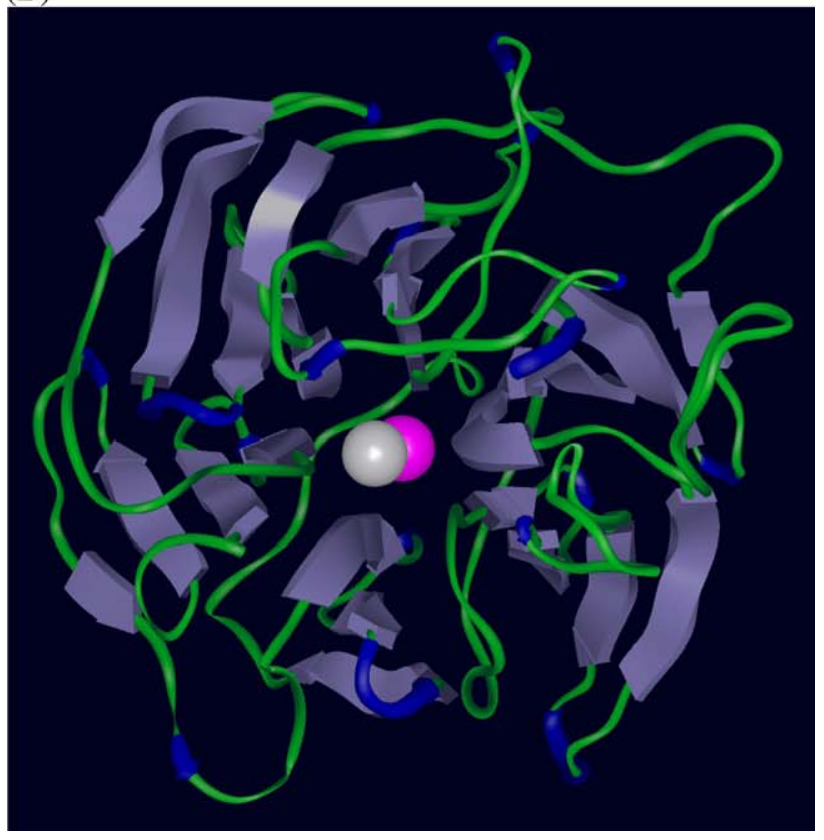

Fig. 1. (A) View of a ribbon diagram of the DFPase structure as determined from X-ray crystallography experiments [23]. (B) Ribbon diagram representation of the proposed secondary structure of HuPON1 viewed along the axis with the catalytic calcium (white) above the structural calcium (pink). The model displays a slightly distorted six-bladed $\beta$ propeller shown in the same relative orientation as (A). HuPON1 was simulated using explicitly solvated dynamics with Insight/Discover software from Accelrys. Computational resources were provided by the National Cancer Institute's Advanced Biomedical Computing Center in Frederick, Maryland.

PON1 crystal structure and the activities displayed by the mutant HuPON1 proteins described here are consistent with our proposed structural model of HuPON1 as a sixfold $\beta$-propeller. 


\section{Materials and methods}

\subsection{Purification of native HuPON1}

HuPON1 was purified from serum as described [23] with minor modifications. In brief, serum was incubated overnight at $4{ }^{\circ} \mathrm{C}$ with $1000-\mathrm{ml}$ Cibacron Blue agarose (Sigma-Aldrich, St. Louis, MO) in $50 \mathrm{mM} \mathrm{Tris-Cl,} 1$ $\mathrm{mM} \mathrm{CaCl} 2,4 \mathrm{M} \mathrm{NaCl}, \mathrm{pH}$ 8.0. After washing with buffer, HuPON1 was eluted with $0.1 \%$ Triton X-100 (TX-100), 0.1\% deoxycholate, $50 \mathrm{mM}$ Tris-Cl, $1 \mathrm{mM}$ $\mathrm{CaCl}_{2}, \mathrm{pH}$ 8.0. Fractions containing active HuPON1 were loaded at $1.5 \mathrm{ml} / \mathrm{min}$ onto a 50-ml DEAE Sepharose anion exchange column and eluted using a $1 \mathrm{ml} / \mathrm{min}$ linear gradient of 0.07 to $0.35 \mathrm{M} \mathrm{NaCl}$. Fractions containing HuPON1 were desalted in a 500-ml gradient by membrane dialysis using a $6-8-\mathrm{kDa}$ molecular weight (MW) cutoff membrane (Spectrum Laboratory, Rancho Dominguez, CA). Samples were dialyzed into $0.1 \%$ TX100, $25 \mathrm{mM}$ Tris-Cl, $1 \mathrm{mM} \mathrm{CaCl}_{2}, \mathrm{pH} 8.0$, and concentrations of HuPON1 were determined by bicinchoninic acid (BCA) assay (Sigma-Aldrich). SDS-PAGE and enzymatic activity against phenyl acetate were performed to estimate purity and specific activity.

\subsection{Concentrating purified HuPON1}

Two methods were utilized to concentrate pure native HuPON1 for CD experiments. First, centrifugal dialysis using Amicon ultra filters with a $10-\mathrm{kDa}$ MW cutoff (Millipore, Billerica, MA) was used to concentrate samples to $450 \mu \mathrm{g} / \mathrm{ml}$ in $4 \mathrm{mM}$ Tris- $\mathrm{Cl}, 1 \mathrm{mM} \mathrm{CaCl}$, $\mathrm{pH} \quad 8.0$ in a final TX-100 concentration that was estimated to be between $0.1 \%$ and $1.0 \%$. In other experiments, purified HuPON1 in $0.1 \%$ TX-100, $2 \mathrm{mM}$ Tris-Cl, $1 \mathrm{mM} \mathrm{CaCl}$, $\mathrm{pH} 8.0$, was loaded onto a 1-ml DEAE sepharose column. HuPON1 was eluted in the same buffer supplemented with $0.35 \mathrm{M} \mathrm{NaCl}$; the resulting concentration of HuPON1 was $320 \mu \mathrm{g} / \mathrm{ml}$. The enzyme was then desalted as described above.

\subsection{Detergent exchange}

The light scattering properties of TX-100 make it an experimentally challenging candidate for $\mathrm{CD}$ experiments. However, octyl $\beta$-D-glucopyranoside (BOG) has been shown to be a more appropriate detergent for CD studies $[29,30]$. To facilitate collection of CD data, we exchanged TX-100 for BOG by loading purified HuPON1 in $0.1 \%$ TX-100, $2 \mathrm{mM}$ Tris-Cl, $1 \mathrm{mM} \mathrm{CaCl}$, pH 8.0 onto a DEAE anion exchange column. The column was then equilibrated with four column volumes of buffer containing $0.1 \% \mathrm{v} / \mathrm{v}$ BOG, $8 \mathrm{mM}$ Tris- $\mathrm{Cl}, 1$ $\mathrm{mM} \mathrm{CaCl} 2, \mathrm{pH}$ 8.0. The protein was finally eluted with the same equilibration buffer containing $0.35 \mathrm{M} \mathrm{NaCl}$, and was desalted as described above.

\subsection{CD spectroscopy}

The CD spectra of purified HuPON1 were recorded at $25{ }^{\circ} \mathrm{C}$ in the presence of TX-100 or BOG using an AVIV 215 spectropolarimeter (Proterion, Piscataway, NJ) with 1$\mathrm{cm}$ pathlength cell. The bandwidth was set at $1 \mathrm{~nm}$, and scans were obtained between 190 and $260 \mathrm{~nm}$ with wavelength increments of either 1.5 or $2.0 \mathrm{~nm}$. For scans of HuPON1 in the presence of TX-100, a stock solution of HuPON1 $(0.32 \mathrm{mg} / \mathrm{ml}(7.6 \mu \mathrm{M})$ in $4 \mathrm{mM}$ Tris- $\mathrm{Cl}$, $0.1 \% \mathrm{TX}-100,1 \mathrm{mM} \mathrm{CaCl} 2, \mathrm{pH} 8.0$ ) was diluted with water to a final concentration of $0.64 \mu \mathrm{M}$. For measurements in $0.1 \%$ BOG, HuPON1 from the enzyme stock solution $(0.23 \mathrm{mg} / \mathrm{ml}(5.48 \mu \mathrm{M})$ in $0.1 \%$ BOG, $8 \mathrm{mM}$ Tris-Cl, $1 \mathrm{mM} \mathrm{CaCl} 2, \mathrm{pH} 8.0$ ) was diluted to $3.0 \mu \mathrm{M}$ in $0.1 \%$ BOG, $8 \mathrm{mM}$ Tris-Cl, $1 \mathrm{mM} \mathrm{CaCl}_{2}, \mathrm{pH}$ 8. Enzyme activity controls were performed to ensure no loss of HuPON1 activity against phenyl acetate. Mean residue ellipticity $[\theta]$ was calculated from the equation $[\theta]=\left(\theta_{\text {obs }}\right)$ $10 L c) / r$, where $\theta_{\text {obs }}$ is the measured ellipticity in millidegrees, $L$ is the pathlength of the cell in $\mathrm{cm}, c$ is the concentration in $\mathrm{M}$, and $r$ is the number of residues.

\subsection{Mutagenesis of HuPON1}

Substitution of amino acid residues in the HuPON1 enzyme was accomplished by PCR-based site-directed mutagenesis of a HuPON1 cDNA template, which also encodes a 6-histidine tag at the $3^{\prime}$ end, cloned into the vector pcDNA3 (Invitrogen, Carlsbad, CA) as described previously [17,31]. Primers used to introduce mutations into HuPON1 cDNA (Table 1) were synthesized using an Oligo 1000 DNA Synthesizer (Beckman Coulter, Fullerton, CA). Mutant forward and reverse gene products were amplified separately and joined together via Gene Splicing by Overlap Extension (gene SOEing [32]) using T7 forward and Sp6 reverse primers external to the gene. Purified PCR products were obtained from a $0.6 \%$ agarose gel using a QIAquick Gel Extraction Kit (Qiagen, Valencia, CA). After purification, products were digested with HindIII and ApaI restriction enzymes (New England BioLabs, Beverly, MA) and ligated into a similarly digested pcDNA3 expression plasmid using T4 ligase (Invitrogen). Ligated products were transformed into electroporation competent DH5- $\alpha$ bacteria (Invitrogen). Bacteria were plated on LB plates containing $100 \mathrm{ig} / \mathrm{ml}$ ampicillin (LB/Amp) to select for ampicillin resistance. Colonies were selected and grown in culture tubes with $3 \mathrm{ml}$ of $\mathrm{LB} / \mathrm{Amp}$ media overnight at $37{ }^{\circ} \mathrm{C}$, and plasmid DNA was purified using Qiagen plasmid mini-prep purification kits.

\subsection{Screening of mutant HuPON1 constructs}

Mutant clones were initially screened by SacI (New England BioLabs) digestion to detect the presence of the HuPON1 gene. HuPON1-containing plasmid clones were 
Table 1

Primers used to introduce substitutions into wild-type HuPON1 cDNA via site-directed mutagenesis and gene splicing by overlap extension

\begin{tabular}{|c|c|c|}
\hline $\begin{array}{l}\text { Wild-type } \\
\text { HuPON1 residue }\end{array}$ & Mutant(s) & Site-directed mutation \\
\hline G11 & $\mathrm{A} / \mathrm{C} / \mathrm{S} *$ & 5'AGACCGGAAGCTTCCGCCAGCCATGGCGAAGCTGATTGCGCTCACCCTCTTG $(\boldsymbol{G} / \boldsymbol{T})(\boldsymbol{G} / \boldsymbol{C})$ TATGGGACT $3^{\prime}$ \\
\hline D54 & $\mathrm{N}$ & 5' ACTGGCTCTGAA $\boldsymbol{A} A C$ ATGGAGATAC $3^{\prime}$ \\
\hline L69 & $\mathrm{F}$ & 5' TAGCTCTGGATT $\overline{T A A G T A T C C T G ~ 3 '}$ \\
\hline H115 & $\mathrm{W}$ & $5^{\prime}$ ATTTAACCCT $\underline{\boldsymbol{T} \boldsymbol{G} \overline{\boldsymbol{G}}}$ GGGATTAGCACA $3^{\prime}$ \\
\hline N133 & $\mathrm{S}$ & $5^{\prime}$ CCTGGTGGTG $\overline{A G C}$ CATCCAGATG $3^{\prime}$ \\
\hline H134 & $\mathrm{W} / \mathrm{Y}$ & 5' GTGGTGAAC $\boldsymbol{T}(\overline{\boldsymbol{A}} / \boldsymbol{G})(\boldsymbol{T} / \boldsymbol{G}) \mathrm{CCAGATG} 3^{\prime}$ \\
\hline N168 & $\mathrm{E}$ & 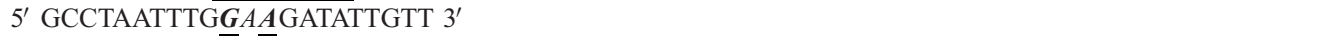 \\
\hline $\mathrm{F} 222$ & $\mathrm{Y}$ & $5^{\prime}$ GAAGGGTTTGATT $\boldsymbol{A}$ TGCTAATGGA $3^{\prime}$ \\
\hline F222 & $\mathrm{A} / \mathrm{D}$ & $5^{\prime}$ GAAGGGTTTGAT $\left.\overline{\boldsymbol{G}} \boldsymbol{A} / \boldsymbol{C}\right) \boldsymbol{C G C T A A T G G A ~} 3^{\prime}$ \\
\hline N224 & A & 5' GATTTTGCT $\boldsymbol{G} \boldsymbol{C}$ T $\overline{\mathrm{GGAAT}} \mathrm{AAACAT} 3^{\prime}$ \\
\hline D269 & $\mathrm{E}$ & $5^{\prime}$ ACCCTCGT $\underline{\boldsymbol{G} \boldsymbol{A} \boldsymbol{A}}$ AACATATCT $3^{\prime}$ \\
\hline $\mathrm{C} 284$ & $\mathrm{D}$ & $5^{\prime}$ TGGGTTGGA $\overline{\underline{\boldsymbol{G} \boldsymbol{A}}} T C A T C C 3^{\prime}$ \\
\hline $\mathrm{H} 285$ & $\mathrm{D} / \mathrm{Y}$ & $5^{\prime}$ TGGATGC $(\boldsymbol{G} / \overline{\boldsymbol{T}}) A T C C C A A T G ~ 3^{\prime}$ \\
\hline V304 & A & 5' GCATCAGAG $\overline{\boldsymbol{T}} \boldsymbol{A} G \mathrm{CTTCGAATC} 3^{\prime}$ \\
\hline E313 & A & $5^{\prime}$ CATTCTAACAG $\underline{C} A$ GAACCTAAAG $3^{\prime}$ \\
\hline E314 & A & $5^{\prime}$ TCTAACAGAA $\bar{G} \underline{\boldsymbol{C}} A$ CCTAAAGTGA $3^{\prime}$ \\
\hline
\end{tabular}

Wild-type and mutant amino acid residues (as indicated by single letter amino acid code) and their positions in the HuPON1 are shown, along with the nucleotide sequences of the primers synthesized for use in mutagenesis experiments. To substitute amino acid residues, nucleotide(s) (bold, underlined) in the targeted codon (italicized) were selectively replaced. Mutating primers were synthesized as shown above, as were their reverse complements; both forward and reverse mutating primers were used in independent polymerase chain reactions with Sp6 and T7 primers, respectively, to generate forward and reverse mutant fragments of wild-type HuPON1 cDNA. These fragments were used in subsequent gene SOEing reactions to generate full-length mutated products.

* The complement of this primer was not synthesized because the residue was sufficiently close to the beginning of the gene that gene SOEing was not necessary. The mutant PON1 gene was generated with the synthesized primer (which included an external T7 primer sequence) and Sp6 primer as the reverse primer.

nucleotide sequenced with BigDye ${ }^{\circledR}$ Version 2.0 (Applied Biosystems, Foster City, CA). Sequencing reactions were run on an ABI Prism 310 Genetic Analyzer and analyzed with Sequence Analyzer Version 1.0.3 and AutoAssembler Version 2.1 (Applied Biosystems). Cloned mutants with the appropriate sequence were then propagated in $120 \mathrm{ml}$ of LB/ Amp and purified using a plasmid maxi kit (Sigma-Aldrich).

\subsection{Transient expression of recombinant HuPON1 wild- type and mutants}

HuPON1-containing pcDNA3 plasmids were transfected into $70-80 \%$ confluent $293 \mathrm{~T}$ human embryonic kidney cells, kindly provided by Dr. O. Lockridge (Eppley Institute, University of Nebraska Medical Center, Omaha, NE), following the protocol of Takagi [33] with slight modifications. Specifically, cells in Dulbecco's Minimal Essential Medium (DMEM, Invitrogen) with $5 \%$ fetal calf serum (Invitrogen) were transfected with 10 $\mu \mathrm{g}$ of HuPON1 mutant DNA in a calcium phosphate transfection solution for $2-3 \mathrm{~h}$. Each mutant construct was independently transfected three times. Secreted protein was harvested 6 days after transfection.

\subsection{SDS-PAGE and Western blotting}

Wild-type and mutant HuPON1 samples were run on $10 \%$ precast TrisGlycine-SDS gels (Invitrogen), and gels were subsequently electroblotted onto Immobilon-P PVDF membrane (Millipore). Membranes were probed with a monoclonal mouse anti-HuPON1 antibody (kindly provided by Dr. R. James, University Hospital of Geneva, Switzerland) at 1:5000 dilution, followed by secondary goat anti-mouse alkaline-phosphatase (AP) conjugated antibody at 1:1000 (Sigma-Aldrich), and incubated at 4 ${ }^{\circ} \mathrm{C}$ overnight. AP activity was detected using BCIP/NBT (Sigma-Aldrich), and images were captured on a Kodak

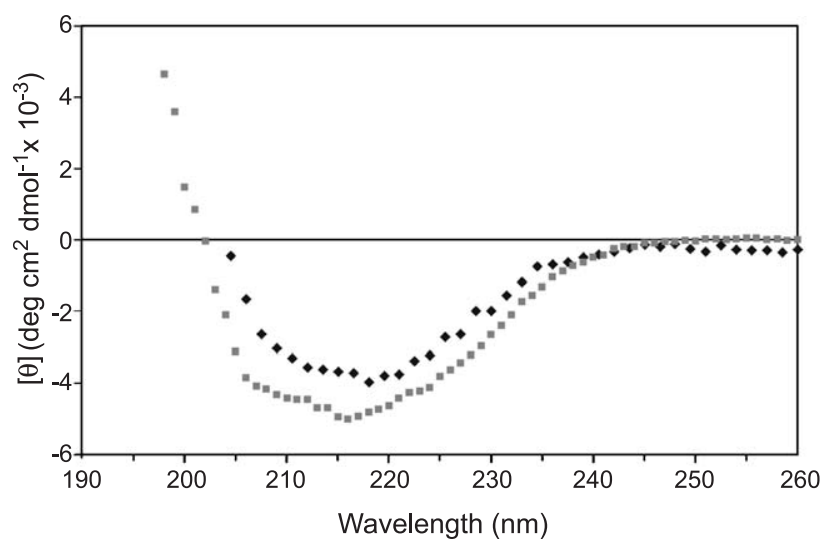

Fig. 2. CD spectra of HuPON1 purified from serum in the presence of either TX-100 or BOG at $25^{\circ} \mathrm{C}$. The spectra of HuPON1 were plotted to exclude low wavelength data prone to error due to light scattering of the TX-100 or BOG detergents. The data were plotted over a range of wavelengths that gave an error of less than $25 \%$. Gray squares: from $198-260 \mathrm{~nm}, 3.0 \mu \mathrm{M}$ HuPON1 in $0.1 \%$ BOG, or Black diamonds: from 205-260 nm, $0.64 \mu \mathrm{M}$ HuPON1 in $0.01 \%$ TX-100. The mean residue ellipticity reported has been corrected for molar protein concentration, path length and amino acid residue in HuPON1 as described in Materials and methods. 


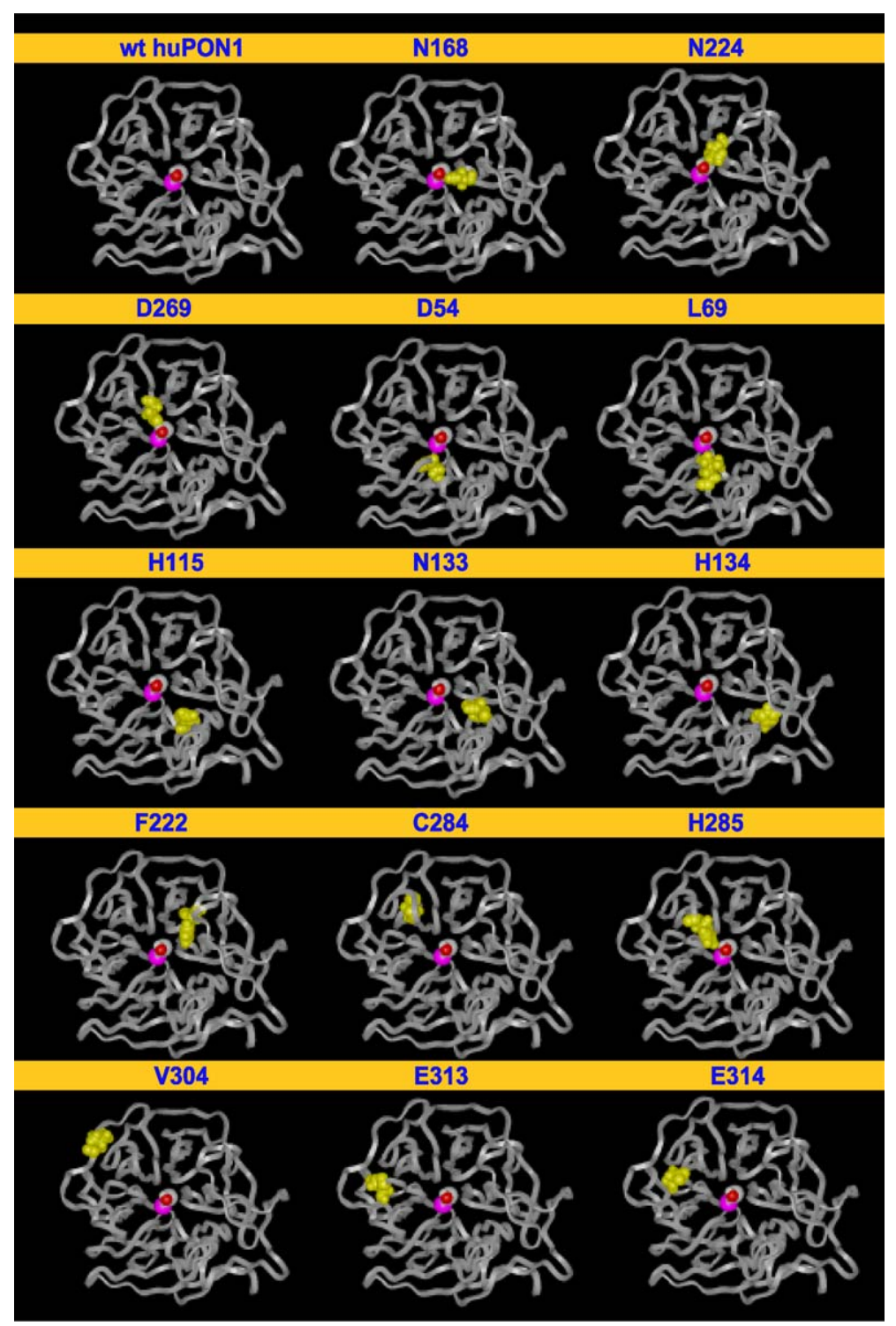

Fig. 3. Graphical depictions of residues selected for mutation (in yellow, as space-filling representations) and their relative locations in the ribbon backbone of the proposed HuPON1 structure are shown. The theoretical catalytic calcium is shown in white along with a modeled water molecule (red and gray), and the proposed structural calcium is shown in pink.

Image Station 440CF (Eastman Kodak, Rochester, NY). Native HuPON1 purified from serum was run on each gel as a positive control.

\subsection{Arylesterase activity}

Arylesterase activity was determined using phenyl acetate (Sigma-Aldrich) in $20 \mathrm{mM}$ Tris- $\mathrm{HCl}$ buffer $\mathrm{pH}$ 8.0 , containing $1 \mathrm{mM} \mathrm{CaCl} 2$, as previously described [19]. Phenyl acetate was used at six concentrations from 0.26 to $3.3 \mathrm{mM}$; if no activity was detected at $3.3 \mathrm{mM}$ for a particular mutant, lower concentrations were not tested. The initial rates of reaction were determined spectrophotometrically by following formation of phenol $\left(\varepsilon=1310 \mathrm{M}^{-1} \mathrm{~cm}^{-1}\right)$ at $A_{270}$ using a Cary $300 \mathrm{BIO} \mathrm{UV/}$ VIS spectrophotometer (Varian, Walnut Creek, CA) at 25
${ }^{\circ} \mathrm{C}$ for 1 min with data gathered at 12 -s intervals. Data were analyzed using Cary WinUV Software Version 2.0 (Varian).

\subsection{Paraoxonase activity}

OP-hydrolase activity was determined using $0.26-2.6$ $\mathrm{mM}$ paraoxon (diethyl $p$-nitrophenyl phosphate; SigmaAldrich) in $50 \mathrm{mM}$ Glycine $/ \mathrm{NaOH}$ buffer $\mathrm{pH} 10.5$, containing $1 \mathrm{mM} \mathrm{CaCl}_{2}[34,35]$. If no activity was detected at $2.6 \mathrm{mM}$ for a particular mutant, lower concentrations were not tested. Initial rates of $p$-nitrophenol formation $\left(\varepsilon=18,290 \mathrm{M}^{-1} \mathrm{~cm}^{-1}\right)$ were followed at $A_{412}$ with a SpectraMax Plus spectrophotometer (Molecular Devices, Sunnyvale, CA) for $10 \mathrm{~min}$. Data were analyzed using Softmax Pro Version 3.0 (Molecular Devices). 


\subsection{Determination of the catalytic parameters}

The catalytic parameter $K_{\mathrm{m}}$ of mutant and wild-type HuPON1 for phenyl acetate and paraoxon was calculated by fitting initial rates of substrate hydrolysis minus spontaneous hydrolysis rates (assessed using supernatant from cells transfected with empty pcDNA3 vector) at six substrate concentrations, when detectable, onto a hyperbolic curve using GraphPad Prism version 3.02 for Windows (GraphPad Software, San Diego, CA) to determine Michaelis-Menten constants. Values for $K_{\mathrm{m}}$ and relative values for $V_{\text {max }}$ were determined for supernatants from at least three independent transfections of each HuPON1 construct. Since only small quantities of the mutant enzymes were produced, we were unable to accurately determine recombinant HuPON1 concentrations. Therefore, only $K_{\mathrm{m}}$ values were calculated from the kinetic data.

\section{Results}

\subsection{HuPON1 purified from human serum}

Starting from a typical prep of $300 \mathrm{ml}$ of human serum, $2.5 \mathrm{mg}$ of purified HuPON1 at a concentration of $0.5 \mathrm{mg} / \mathrm{ml}$ was obtained. In agreement with past results [28], the specific activity of the purified HuPON1 fractions against phenyl acetate was $\sim 1500 \mathrm{U} / \mathrm{mg}$.

\subsection{Secondary structure of HuPON1 as deduced by $C D$ spectroscopy}

The CD spectra presented in Fig. 2 show curves for HuPON1 in the presence of the detergents TX-100 or BOG. Light scattering resulting from the presence of detergents complicated the accurate measurement of the CD spectra of HuPON1. TX-100, with a low critical micelle concentration (CMC) value of $0.2-0.9 \mathrm{mM}$, has a significant micelle population at concentrations of the detergent that are
(A)

Arylesterase Activity

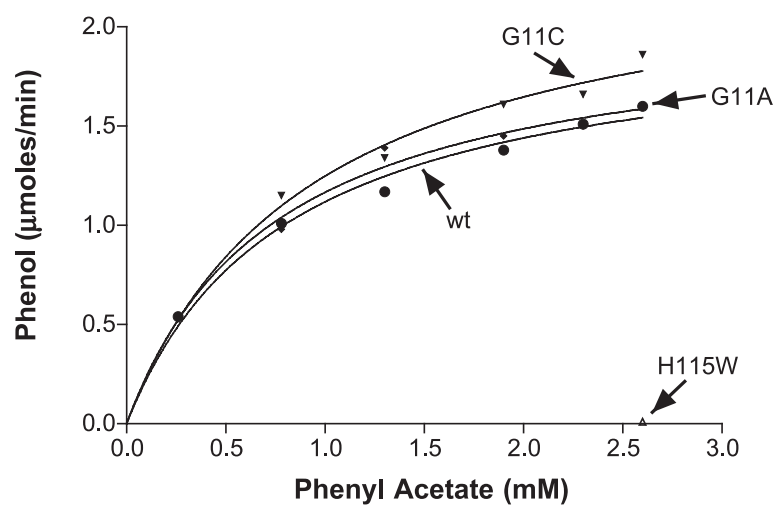

(B) Paraoxonase Activity

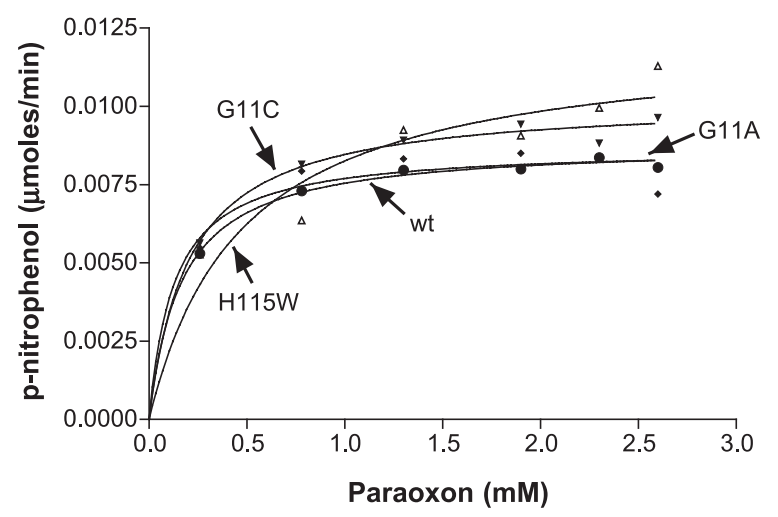

Fig. 5. Michaelis-Menten plots for the hydrolysis of phenyl acetate (A) and paraoxon (B) by recombinant wild-type HuPON1 (๑) and representative variants G11A $(\diamond)$, G11C $(\nabla)$ and $\mathrm{H} 115 \mathrm{~W}(\triangle)$. Data shown are representative of at least three independent experiments. The kinetic parameter $K_{\mathrm{m}}$ derived from the fits is presented in Table 2. Measurements and analyses of arylesterase and paraoxonase activities are detailed in Materials and methods.

necessary for solubilizing and maintaining the long-term stability of HuPON1. To help reduce micelle-induced light scattering, CD data were collected following the dilution of HuPON1 in TX-100 from $0.1 \%$ to about $0.01 \%$ (reducing

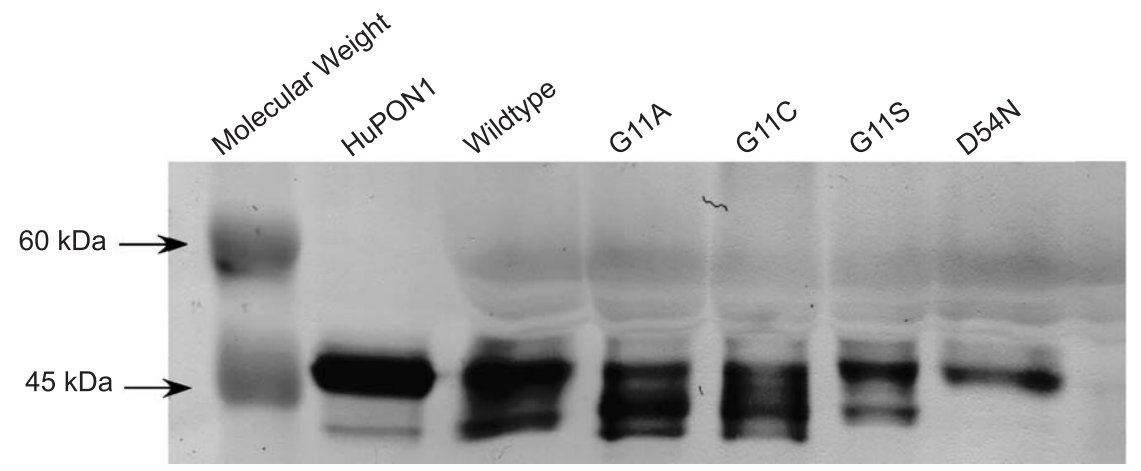

Fig. 4. Western blot of purified native HuPON1, recombinant wild-type and selected mutants. Each lane was loaded with $40 \mu 1$ of recombinant HuPON1 in culture medium and SDS Laemmli sample buffer $(1: 1 \mathrm{v} / \mathrm{v})$. Samples were transferred to PVDF membrane and probed with a monoclonal mouse anti-HuPON1 antibody and probed with alkaline-phosphatase conjugated rabbit anti-mouse antibody. Images were developed via BCIP/NBT liquid substrate system and visualized by scanning. Comparable blots were obtained with mutants L69F, H115W, N133S, H134W, H134Y, N168E, F222D, F222Y, N224A, D269E, C284D, H285D, H285Y, V304A, E313A, E314A, and the double mutant H115W/N133S (data not shown). 
Table 2

Amino acid residues predicted to play a functional role in HuPON1 substrate binding and catalysis

\begin{tabular}{|c|c|c|c|c|c|}
\hline \multirow[t]{2}{*}{ Predicted role } & \multirow[t]{2}{*}{ reHuPON1 } & \multicolumn{2}{|c|}{ Phenyl acetate } & \multicolumn{2}{|l|}{ Paraoxon } \\
\hline & & $K_{\mathrm{m}}(\mathrm{mM})^{\mathrm{a}}$ & $\% K_{\mathrm{m}}$ to $\mathrm{wt}^{\mathrm{b}}$ & $K_{\mathrm{m}}(\mathrm{mM})^{\mathrm{a}}$ & $\% K_{\mathrm{m}}$ to $\mathrm{wt}^{\mathrm{b}}$ \\
\hline \multirow[t]{3}{*}{ N-terminus signaling peptide } & G11A & 0.74 & 121 & 0.18 & 113 \\
\hline & $\mathrm{G} 11 \mathrm{C}$ & 0.74 & 121 & 0.19 & 119 \\
\hline & G11S & 0.64 & 105 & 0.18 & 113 \\
\hline \multirow[t]{3}{*}{ Catalytic calcium binding } & N168E & nd & - & nd & - \\
\hline & N224A & nd & - & nd & - \\
\hline & D269E & nd & - & nd & - \\
\hline Structural calcium binding & D54N & nd & - & nd & - \\
\hline \multirow[t]{10}{*}{ Substrate binding site } & L69F & nd & - & nd & - \\
\hline & H115W & nd & - & 0.42 & 262 \\
\hline & N133S & 0.59 & 97 & 0.17 & 106 \\
\hline & H115W/N133S & nd & - & 0.15 & 83 \\
\hline & H134W & nd & - & nd & - \\
\hline & H134Y & nd & - & nd & - \\
\hline & F222D & nd & - & nd & - \\
\hline & $\mathrm{F} 222 \mathrm{Y}$ & 0.89 & 146 & nd & - \\
\hline & N224A & nd & - & nd & - \\
\hline & C284D & nd & - & nd & - \\
\hline \multirow[t]{2}{*}{ Catalytic site } & H285D & nd & - & nd & - \\
\hline & $\mathrm{H} 285 \mathrm{Y}$ & nd & - & nd & - \\
\hline \multirow[t]{3}{*}{ Surface residue } & E313A & 0.51 & 84 & 0.14 & 88 \\
\hline & E314A & 0.67 & 106 & 0.19 & 119 \\
\hline & V304A & nd & - & nd & - \\
\hline
\end{tabular}

nd=not detectable at 3.3 and $2.6 \mathrm{mM}$ substrate concentrations for phenyl acetate and paraoxon, respectively.

${ }^{\text {a }} K_{\mathrm{m}}$ values are averages of at least three experiments with supernatants from independent transfections.

b Relative to recombinant wild-type $K_{\mathrm{m}}$ values for phenyl acetate $\left(K_{\mathrm{m}}=0.61 \mathrm{mM}\right)$ and paraoxon $\left(K_{\mathrm{m}}=0.16 \mathrm{mM}\right)$ as substrates, respectively.

the TX-100 concentration from $\sim 1.5$ to $\sim 0.15 \mathrm{mM}$ ). Additionally, HuPON1 was exchanged into the detergent BOG, which has a higher CMC (20-25 mM) and therefore greatly reduced light scattering. Despite these improvements, the $\mathrm{CD}$ data were deemed reliable only down to a wavelength of $198 \mathrm{~nm}$ for the HuPON1/BOG sample. Also noteworthy, the CD spectra are comparable for HuPON1 in either $0.01 \%$ TX-100 or BOG. This result is consistent with activity assays that showed the enzyme retained full activity in $0.01 \% \mathrm{TX}-100$ for roughly $30 \mathrm{~min}$ (data not shown). The $\mathrm{CD}$ data presented in Fig. 2 are reported in units of mean residue ellipticity. The most informative analyses were obtained by comparing the HuPON1 CD spectra with published CD spectra of other known $\beta$-fold proteins. The HuPON1 CD spectra, with a broad minimum centered around $217 \mathrm{~nm}$ and a crossover point of $205 \mathrm{~nm}$, are consistent with a mainly $\beta$-sheet protein fold [36].

\subsection{Design of mutant HuPON1 enzymes}

Based on previous studies [17,22], we chose to alter selected amino acid residues (glycine, aspartate, cysteine, histidine, leucine, asparagine, glutamate, phenylalanine and valine), some predicted to be critical for HuPON1's arylesterase and organophosphatase enzymatic activities (Fig. 3). Targeted residues were predicted from our model to be in the catalytic site (H285) or substrate binding sites (L69, H115, N133, H134, H184, F222, and C284). Possible ligands of calcium ions (D54, N168, N224, and
D269) were also targets of mutagenesis, as were the solvent-exposed residues V304, E313 and E314. Residue G11 in the N-terminus signaling peptide was substituted, even though the signal peptide region did not align with any residues in DFPase, and thus is not modeled in the theoretical structure [19]. The relative locations of the altered residues in the mutants are shown superimposed on the proposed secondary structure of wild-type HuPON1 (Fig. 3; [19]). As a negative control, an empty pcDNA3 vector was also designed.

\subsection{Expression of recombinant wild-type and mutant HuPON1}

Vectors encoding recombinant wild-type HuPON1, mutant HuPON1s, HuBuChE, and no gene (empty pcDNA3 vector) were transiently transfected into $293 \mathrm{~T}$ cells. Expression of secreted protein in the cell culture supernatants was detected by Western blot using a HuPON1-specific monoclonal antibody. Supernatants from wild-type HuPON1 served as positive controls, whereas supernatants from empty pcDNA3 and HuBuChE-transfected cells were negative controls. Recombinant HuPON1 molecules comigrated with purified native HuPON1 $(38-43 \mathrm{kDa})$, as shown in Fig. 4. Consistent with past findings [27], recombinant HuPON1 proteins migrated as either a single band, a doublet, or a triplet, depending on glycosylation state [37,38]. All of the mutant enzymes were expressed at concentrations that were easily detectable by Western blot 
and no bands were detected from either $\mathrm{HuBuChE}$ or empty pcDNA3 control supernatants.

\subsection{Enzymatic analysis of recombinant HuPON1 proteins}

$K_{\mathrm{m}}$ values of active mutants were compared with those of the recombinant wild-type HuPON1 to determine relative affinities (Fig. 5A and $\mathrm{B}$ for representative results; summarized in Table 2). Rates of spontaneous hydrolysis of the substrates in arylesterase and paraoxonase assays were accounted for using supernatants from cells transfected with empty vector and/or HuBuChE; these background rates were subtracted from each sample to yield net rates of hydrolysis. Loss of arylesterase and paraoxonase activities (defined as undetectable enzymatic activities at $3.3 \mathrm{mM}$ and $2.6 \mathrm{mM}$ phenyl acetate and paraoxon concentrations, respectively) was observed in all mutants except G11, E313, E314, H115, N133 and F222. Both the H115W mutant and the H115W/N133S double mutant had no detectable arylesterase activity, but retained paraoxonase activity (with a 2.5 -fold increase in $K_{\mathrm{m}}$ for H115W). Conversely, F222Y retained the ability to hydrolyze phenyl acetate with a $\sim 50 \%$ increase in its $K_{\mathrm{m}}$ relative to wild-type, but had no detectable paraoxonase activity. Interestingly, the F222D mutant resulted in complete loss of activity against both phenyl acetate and paraoxon. Unexpectedly, V304A had no detectable ability to hydrolyze either phenyl acetate or paraoxon.

\section{Discussion}

In the present study, we sought to verify a homology model of HuPON1 by CD spectroscopy and site-directed mutation analyses to identify residues predicted to be critical for enzymatic activity. Our homology model suggests that HuPON1 adopts a six-bladed $\beta$-propeller fold similar to the DFPase protein structure, on which our theoretical structural model is based.

\subsection{CD spectroscopy}

The CD spectrum of HuPON1 (Fig. 2) is comparable to that of a published DFPase CD spectrum [39] reported to have $49 \% \beta$-sheet, $16 \% \beta$-turns and 35\% random coils with no $\alpha$-helical structure. Analysis of our HuPON1 spectrum with the Definition of Secondary Structure of Protein (DSSP, http://www.cmbi.kun.nl/gv/dssp/) indicates that it is $43 \% \beta$-sheeted, $15 \% \beta$-turns, and $34 \%$ random coils, and $8 \% \quad \alpha$-helical. The broad HuPON1 CD minimum at $217 \mathrm{~nm}$ is similar to the minimum at 209 $\mathrm{nm}$ observed in DFPase. It is noteworthy that the HuPON1 minimum at $217 \mathrm{~nm}$ and the crossover at a wavelength of $203 \mathrm{~nm}$ for HuPON1/BOG are in very close agreement with the position of the standard CD spectrum of $\beta$-sheet peptides and proteins, which gen- erally have a minimum at $217 \mathrm{~nm}$ and crossover points near $205 \mathrm{~nm} \mathrm{[36].}$

\subsection{Mutation in the N-terminal signal peptide}

The N-terminal signal peptide of HuPON1 includes the first 40 amino acids of the expressed protein. Residues L9 to F17 constitute the hydrophobic core of this leader sequence $[9,40]$. The retention of this peptide in the mature protein [41], determined by direct sequencing of purified HuPON1, led to the conclusion that this highly hydrophobic region is involved in HDL binding in vivo, which was subsequently confirmed by Sorenson et al. [9]. We hypothesize that HDL binding in vivo is mediated by an $\alpha$-helical trans-membrane anchor adopted by the N-terminal signal peptide [42]. To test this hypothesis, we attempted to disrupt the formation of the $\alpha$-helix by substituting residue G11 to determine effects on enzymatic function. The residue was replaced with similarly sized amino acid residues (serine, cysteine, or alanine), with different functional groups (-OH, - $\mathrm{SH}$ or $\mathrm{CH}_{3}$, respectively), with no observable effect on enzyme function. This indicates that the N-terminal signal peptide does not have an active role in HuPON1 catalysis [9] and further suggests that this position is not directly linked to the active site structure or enzymatic mechanism. The result is consistent with our hypothesis, which predicts that this residue is part of a HDL-anchoring helix and, as such, mutations at this position are not expected to effect protein structure directly.

\subsection{Mutating residues predicted to bind the structural or catalytic calcium ions}

HuPON1 has an absolute requirement for calcium as a cofactor for substrate binding and catalysis [37,38]. It is worthwhile to note that calcium-binding residues can be dispersed anywhere throughout the primary sequence $[19,38]$. Alteration of the structural calcium ligand D54 and the catalytic calcium ligands N168, N224 and D269 ablated detectable enzyme activity at the highest substrate concentrations of phenyl acetate $(3.3 \mathrm{mM})$ and paraoxon $(2.6 \mathrm{mM})$. This suggests that the residues predicted to be involved in calcium binding are critical for enzymatic activities. Changing an aspartate to asparagine at residue 54, predicted to be important for binding the structural calcium, eliminated enzymatic activity of the resulting HuPON1 mutant, although the protein was sufficiently folded and stable to be detected by Western blot in the 293T cell supernatant. Likewise, the substitution of residues N168, $\mathrm{N} 224$, or D269, proposed to be involved in the binding of the catalytic calcium ion, with glutamate abolished all enzymatic activities. The N168E mutation resulted in the insertion of a residue with an increased size and a negative charge, N224A replaced a polar residue with a small nonpolar residue, and D269E conserved the charge but increased the size of the residue by a methylene group. In 
our model, residues N168, N224, and D269 are solventaccessible, suggesting that small alterations in the amino acid side chains at these positions may cause perturbations in surrounding residues and/or the size and orientation of the calcium binding site. Since N168E, N224A, and D269E substitutions dramatically affected HuPON1 activity, it is likely that the catalytic calcium-binding site in this structure is conformationally inflexible.

\subsection{Disrupting predicted surface residues}

Mutating residues at positions 313 and 314 did not affect enzymatic function of HuPON1, consistent with predictions from our model that place these residues on the enzyme's surface at solvent-exposed sites. Interestingly, when residue V304, which also is predicted to be a solvent-exposed amino acid in our structural model, was replaced with alanine, enzymatic activity was lost. The reason for this loss is unclear; it is possible that this mutation either disrupts proper folding of HuPON1 or alters association of the mutant enzyme with associated lipoproteins. As was noted for the D54N mutant, V304A was easily detected by Western blot, suggesting that the substituted protein adopts a conformation that allows it to be secreted by $293 \mathrm{~T}$ cells and retained in the culture supernatant. Additional substitutions of this residue are planned.

\subsection{Mutation of putative substrate binding residues}

Replacement with an aromatic ring at position L69 ablated detectable enzymatic activity against both phenyl acetate and paraoxon. This suggests that the size of the residue at this position may be critical for substrate binding to occur. Substitution of tryptophan or tyrosine at position H134 also abolished HuPON1's ability to catalyze the hydrolysis of both phenyl acetate and paraoxon. These results highlight the importance of residues L69 and H134 for phenyl acetate and paraoxon binding. In contrast, the replacement of H115 with tryptophan eliminated arylesterase but not paraoxonase activity. This substitution also increased the $K_{\mathrm{m}}$ for paraoxon by more than twofold. Interestingly, an unintended PCR-induced double mutant of HuPON1 with both H115W and N133S substitutions had a $K_{\mathrm{m}}$ for paraoxon that was indistinguishable from that of wild-type HuPON1, but had no detectable arylesterase activity. The increase in $K_{\mathrm{m}}$ for $\mathrm{H} 115 \mathrm{~W}$ could be due to the steric effects of the larger tryptophan residue preventing paraoxon from accessing the catalytic site of the enzyme. The subsequent replacement of the nearby N133 with a smaller serine residue in the double mutant might offset the steric constraints placed by the aromatic tryptophan at position 115. Interestingly, when the N133S mutation is expressed alone, no effect on either arylesterase or paraoxonase activity was observed. Taken together, these results suggest that residue $\mathrm{H} 115$ is not directly involved in the catalytic mechanism of HuPON1, but rather that it most likely resides near, if not within, the catalytic machinery and functions as a substrate-selective residue. This conclusion is in disagreement with that of Harel et al. [20], who postulated, based on the crystal structure of a hybrid PON1 enzyme, that H115 couples with H134 to create a catalytic dyad at the active site of the enzyme. It is likely that analysis of additional histidine substitutions will help resolve this issue.

In a similar vein, the conservative substitution of phenylalanine at residue 222 with tyrosine did not ablate arylesterase activity but eliminated paraoxonase activity. However, substitution of the same residue to aspartate abolished both arylesterase and organophosphatase activity. Coupled with our H115 findings, the F222 mutation results suggest that HuPON1 either has differential binding requirements for different substrates, as was suggested by Harel et al. [20], or has more than one catalytic site. HuPON1 and HuPON3 share a high degree of homology in amino acid sequence $[37,43,44]$, yet they have very different arylesterase versus paraoxonase activities. It is worth noting that, while H115 is found in both isozymes, serine is found at position 222 in HuPON3 instead of phenylalanine [20]. It is likely that the amino acid residues at positions 115 and 222 play a role in defining the catalytic differences and/or substrate specificities between the two isozymes $[25,45]$.

\subsection{Mutating possible catalytic residues}

Mutation of the H285 residue by substitution to either tyrosine or aspartate eliminated all enzymatic activity, consistent with previous results [19], indicating that this residue is essential for catalytic activity. As a complement to these results, we replaced the adjacent C284 with aspartate (C284D). Prior results [46] postulated that C284 plays a role in substrate binding but is not essential for arylesterase activity. However, in the crystal structure of the PON1 hybrid enzyme, C284 does not appear to have a functional role at all since it is deeply buried within the protein and probably serves only to stabilize the core structure [20]. In our study, the mutation C284D eliminated HuPON1's catalytic activity against phenyl acetate and paraoxon. We postulate that the introduction of a negatively charged residue at this position eliminated catalytic activity by causing a change in the electrostatics and/or positioning of the adjacent $\mathrm{H} 285$ residue, suggesting that $\mathrm{H} 285$ may be directly involved in the catalytic mechanism of HuPON1. Interestingly, residue V304 in the crystal structure resides in close proximity to $\mathrm{C} 284$, but distal from H285 [20]. The possibility that a V304A substitution alters the position of residue C284, which in turn effects $\mathrm{H} 285$, offers a potential explanation for the loss of activity observed in the V304A mutant. The current results do not contradict previous findings and suggest that C284 resides at or very near the enzyme active site. 
The structure of a hybrid PON1 was recently determined at $2.2 \AA$ [20]. Consistent with the HuPON1 structural model presented here, this enzyme was found to be a six-bladed $\beta$-propeller protein akin to DFPase (on which our homology model was based). Although the results of our site-directed mutagenesis experiments at H115 and H134 contradict the hypothesis posed by Harel et al. [20] to describe the catalytic mechanism, in general, our structural model is in very close agreement with the crystal structure. We believe that together with the recently obtained crystal structure, the HuPON1 homology model described here will be a valuable tool for detailed analysis of the mechanisms involved in HuPON1 catalysis and will inform future genetic engineering efforts to enhance the OPase activity of HuPON1.

\section{Acknowledgement}

D.T.Y. was supported by an appointment to the Internship/Research Participation Program for the US Army Medical Research Institute of Chemical Defense, administered by the Oak Ridge Institute for Science and Education through an agreement between the US Department of Energy and the USAMRICD. B.J.B. was supported by NIH Grant 5P20RR015588.

\section{References}

[1] B. Ballantyne, T.C. Marrs, Overview of the biological and clinical aspects of organophosphates and carbamates, in: B. Ballantyne, T.C. Marrs (Eds.), Clinical and Experimental Toxicology of Organophosphates and Carbamates, Butterworth, Oxford, 1992, pp. 1-14.

[2] D.M. Maxwell, C.A. Castro, D.M. De La Hoz, M.K. Gentry, M.B. Gold, R.P. Solana, A.D. Wolfe, B.P. Doctor, Protection of rhesus monkeys against soman and prevention of performance decrement by pretreatment with acetylcholinesterase, Toxicol. Appl. Pharmacol. 115 (1992) 44

[3] L.M. Watkins, H.J. Mahoney, J.K. McCulloch, F.M. Raushel, Augmented hydrolysis of diisopropylfluorophosphate in engineered mutants of phosphotriesterase, J. Biol. Chem. 272 (1997) 25596.

[4] B.P. Doctor, D.W. Blick, G. Caranto, C.A. Castro, M.K. Gentry, R. Larrison, D.M. Maxwell, M.R. Murphy, M. Schutz, K. Waibel, A.D. Wolfe, Cholinesterases as scavengers for organophosphorus compounds: protection of primate performance against soman toxicity, Chem. Biol. Interact. 87 (1993) 285.

[5] C.A. Broomfield, D.M. Maxwell, R.P. Solana, C.A. Castro, A.V. Finger, D.E. Lenz, Protection by butyrylcholinesterase against organophosphorus poisoning in nonhuman primates, J. Pharmacol. Exp. Ther. 259 (1991) 633.

[6] D.E. Lenz, C.A. Broomfield, D.M. Maxwell, D.M. Cerasoli, Nerve Agent Bioscavengers: Protection Against High- and Low-Dose Organophosphorus Exposure, in: J. Romano, S. Somani (Eds.), Chemical Warfare Agents: Toxicity at Low Levels, CRC Press LLC, Boca Raton, 2001, pp. 245-260.

[7] D.M. Cerasoli, D.E. Lenz, Nerve agent bioscavengers: protection with reduced behavioral effects, Mil. Psychol. 14 (2002) 121.

[8] D.M. Shih, L. Gu, Y.R. Xia, M. Navab, W.F. Li, S. Hama, L.W. Castellani, C.E. Furlong, L.G. Costa, A.M. Fogelman, A.J. Lusis, Mice lacking serum paraoxonase are susceptible to organophosphate toxicity and atherosclerosis, Nature 394 (1998) 284.
[9] R.C. Sorenson, C.L. Bisgaier, M. Aviram, C. Hsu, S. Billecke, B.N. $\mathrm{La} \mathrm{Du}$, Human serum paraoxonase/arylesterase's retained hydrophobic N-terminal leader sequence associates with HDLs by binding phospholipids: apoliproprotein A-I stabilizes activity, Arterioscler. Thromb. Vasc. Biol. 19 (1999) 2214.

[10] B. Mackness, G.K. Davies, W. Turkie, E. Lee, D.H. Roberts, E. Hill, C. Roberts, P.N. Durrington, M.I. Mackness, Paraoxonase status in coronary heart disease: are activity and concentration more important than genotype? Arterioscler. Thromb. Vasc. Biol. 21 (2001) 1451.

[11] C.E. Furlong, W.F. Li, L.G. Costa, R.J. Richter, D.M. Shih, A.J. Lusis, Genetically determined susceptibility to organophosphorus insecticides and nerve agents: developing a mouse model for the human PON1 polymorphism, Neurotoxicology 19 (1998) 645.

[12] L.G. Costa, W.F. Li, R.J. Richter, D.M. Shih, A. Lusis, C.E. Furlong, The role of paraoxonase (PON1) in the detoxification of organophosphates and its human polymorphism, Chem. Biol. Interact. 119-120 (1999) 429.

[13] W.F. Li, L.G. Costa, R.J. Richter, T. Hagen, D.M. Shih, A. Tward, A.J. Lusis, C.E. Furlong, Catalytic efficiency determines the in vivo efficacy of PON1 for detoxifying organophosphorus compounds, Pharmacogenetics 10 (2000) 767.

[14] S.A. Akgur, P. Ozturk, I. Solak, A.R. Moral, B. Ege, Human serum paraoxonase (PON1) activity in acute organophosphorus insecticide poisoning, Forensic Sci. Int. 133 (2003) 136.

[15] C.B. Millard, O. Lockridge, C.A. Broomfield, Design and expression of organophosphorus acid anhydride hydrolase activity in human butyrylcholinesterase, Biochemistry 34 (1995) 15925.

[16] O. Lockridge, R.M. Blong, P. Masson, M.T. Froment, C.B. Millard, C.A. Broomfield, A single amino acid substitution, Gly117His, confers phosphotriesterase (organophosphorus acid anhydride hydrolase) activity on human butyrylcholinesterase, Biochemistry 36 (1997) 786.

[17] D. Josse, O. Lockridge, W. Xie, C.F. Bartels, L.M. Schopfer, P. Masson, The active site of human paraoxonase (PON1), J. Appl. Toxicol. 21 (Suppl. 1) (2001) 7.

[18] J.B. Clendenning, R. Humbert, E.D. Green, C. Wood, D. Traver, C.E. Furlong, Structural organization of the human PON1 gene, Genomics 35 (1996) 586.

[19] D. Josse, W. Xie, F. Renault, D. Rochu, L.M. Schopfer, P. Masson, O. Lockridge, Identification of residues essential for human paraoxonase (PON1) arylesterase/organophosphatase activities, Biochemistry 38 (1999) 2816.

[20] M. Harel, A. Aharoni, L. Gaidukov, B. Brumshtein, O. Khersonsky, R. Meged, H. Dvir, R.B. Ravelli, A. McCarthy, L. Toker, I. Silman, J.L. Sussman, D.S. Tawfik, Structure and evolution of the serum paraoxonase family of detoxifying and anti-atherosclerotic enzymes, Nat. Struct. Mol. Biol. 11 (2004) 412.

[21] H.G. Davies, R.J. Richter, M. Keifer, C.A. Broomfield, J. Sowalla, C.E. Furlong, The effect of the human serum paraoxonase polymorphism is reversed with diazoxon, soman, and sarin, Nat. Genet. 14 (1996) 334

[22] D. Josse, C.A. Broomfield, D. Cerasoli, S. Kirby, J. Nicholson, B. Bahnson, D.E. Lenz, Engineering of HuPON1 for a use as a catalytic bioscavenger in organophosphate poisoning, U.S. Army Medical Defense Bioscience Review 2002, U.S. Army Medical Research Institute of Chemical Defense, Aberdeen Proving Ground, MD, 2002, DTIC No.: Pending.

[23] E.I. Scharff, C. Lucke, G. Fritzsch, J. Koepke, J. Hartleib, S. Dierl, H. Ruterjans, Crystallization and preliminary X-ray crystallographic analysis of DFPase from Loligo vulgaris, Acta Crystallogr., D Biol. Crystallogr. 57 (2001) 148.

[24] T. Fujita, T. Shirasawa, N. Maruyama, Isolation and characterization of genomic and cDNA clones encoding mouse senescence marker protein-30 (SMP30), Biochim. Biophys. Acta 1308 (1996) 49.

[25] D.I. Draganov, P.L. Stetson, C.E. Watson, S.S. Billecke, B.N. La Du, Rabbit serum paraoxonase 3 (PON3) is a high density lipoproteinassociated lactonase and protects low density lipoprotein against oxidation, J. Biol. Chem. 275 (2000) 33435. 
[26] S. Billecke, D. Draganov, R. Counsell, P. Stetson, C. Watson, C. Hsu, B.N. La Du, Human serum paraoxonase (PON1) isozyme Q and R hydrolze lactones and cyclic carbonate esters, Drug Metab. Dispos. 28 (2000) 1335 .

[27] L. Rodrigo, B. Mackness, P.N. Durrington, A. Hernandez, M.I. Mackness, Hydrolysis of platelet-activating factor by human serum paraoxonase, Biochem. J. 354 (2001) 1.

[28] K.N. Gan, A. Smolen, H.W. Eckerson, B.N. La Du, Purification of human serum paraoxonase/arylesterase. Evidence for one esterase catalyzing both activities, Drug Metab. Dispos. 19 (1991) 100.

[29] S. Ghobadi, S. Safarian, A.A. Moosavi-Movahedi, B. Ranjbar, Octyl glucoside induced formation of the molten globule-like state of glutamate dehydrogenase, J. Biochem. 130 (2001) 671.

[30] L.M. Brill, S. Dechongkit, B. DeLaBarre, J. Stroebel, R.N. Beachy, M. Yeager, Dimerization of recombinant tobacco mosaic virus movement protein, J. Virol. 78 (2004) 3372.

[31] B. Chen, A.E. Przybyla, An efficient site-directed mutagenesis method based on PCR, BioTechniques 17 (1994) 657.

[32] R.M. Horton, PCR-mediated recombination and mutagenesis: SOEing together tailor-made genes, Mol. Biotechnol. 3 (1995) 93.

[33] Jun Takagi. Transient Transfection into 293T Cells. (2000) Springer Lab Protocol. Ref Type: Electronic Citation http://cbr.med.harvard. edu/investigators/springer/lab/protocols/protocols_list.html.

[34] D.E. Lenz, L.E. Deguehery, J.S. Holton, On the nature of the serum enzyme catalyzing paraoxon hydrolysis, Biochim. Biophys. Acta 321 (1973) 189.

[35] M. Aviram, S. Billecke, R. Sorenson, C. Bisgaier, R. Newton, M. Rosenblat, J. Erogul, C. Hsu, C. Dunlop, B. La Du, Paraoxonase active site required for protection against LDL oxidation involves its free sulfhydryl group and is different from that required for its arylesterase/ paraoxonase activities: selective action of human paraoxonase allozymes Q and R, Arterioscler. Thromb. Vasc. Biol. 18 (1998) 1617.

[36] S.V. Kuznetsov, J. Hilario, T.A. Keiderling, A. Ansari, Spectroscopic studies of structural changes in two beta-sheet-forming peptides show an ensemble of structures that unfold noncooperatively, Biochemistry 42 (2003) 4321
[37] C.L. Kuo, B.N. La Du, Comparison of purified human and rabbit serum paraoxonases, Drug Metab. Dispos. 23 (1995) 935

[38] C.L. Kuo, B.N. La Du, Calcium binding by human and rabbit serum paraoxonases. Structural stability and enzymatic activity, Drug Metab. Dispos. 26 (1998) 653.

[39] J. Hartleib, H. Ruterjans, High yield expression, purification, and characterization of the recombinant diisopropylfluorophosphatase from Loligo vulgaris, Protein Expr. Purif. 21 (2001) 210.

[40] S. Adkins, K.N. Gan, M. Mody, B.N. La Du, Molecular basis for the polymorphic forms of human serum paraoxonase/arylesterase: glutamine or arginine at the position 191, for the respective A or B allozymes, Am. J. Hum. Genet. 52 (1993) 598.

[41] C. Hassett, R.J. Richter, R. Humbert, C. Chapline, J.W. Crabb, C.J. Omiecinski, C.E. Furlong, Characterization of cDNA clones encoding rabbit and human serum paraoxonase: the mature protein reatins its signal sequence, Biochemistry 30 (1991) 10141.

[42] D. Josse, C. Ebel, D. Stroebel, A. Fontaine, F. Borges, A. Echalier, D. Baud, F. Renault, M. Le Maire, E. Chabrieres, P. Masson, Oligomeric states of the detergent-solubilized human serum paraoxonase (PON1), J. Biol. Chem. 277 (2002) 33386.

[43] S.L. Primo-Parmo, R.C. Sorenson, J. Teiber, B.N. La Du, The human serum paraoxonase/arylesterase gene (PON1) is one member of a multigene family, Genomics 33 (1996) 498.

[44] A. Aharoni, L. Gaidukov, S. Yagur, L. Toker, I. Silman, D.S. Tawfik, Directed evolution of mammalian paraoxonase PON1 and PON3 for bacterial expression and catalytic specialization, Proc. Natl. Acad. Sci. U. S. A. 101 (2004) 482.

[45] J.F. Teiber, D.I. Draganov, B.N. La Du, Lactonase and lactonizing activities of human serum paraoxonase (PON1) and rabbit serum PON3, Biochem. Pharmacol. 66 (2003) 887.

[46] R.C. Sorenson, S.L. Primo-Parmo, C.L. Kuo, S. Adkins, O. Lockridge, B.N. La Du, Reconsideration of the catalytic center and mechanism of mammalian paraoxonase/arylesterase, Proc. Natl. Acad. Sci. U. S. A. 92 (1995) 7187. 